\title{
MicroRNA-219 overexpression serves a protective role during liver fibrosis by targeting tumor growth factor $\beta$ receptor 2
}

\author{
LI MA ${ }^{1}$, JIAN MA ${ }^{2}$ and HONG-LIANG OU ${ }^{1}$ \\ ${ }^{1}$ Department of Liver Diseases, Ningbo No. 2 Hospital, Ningbo, Zhejiang 315010; \\ ${ }^{2}$ Department of Endocrinology, The People's Hospital of Fenghua District, Ningbo, Zhejiang 315500, P.R. China
}

Received May 10, 2018; Accepted November 5, 2018

DOI: $10.3892 / \mathrm{mmr} .2018 .9787$

\begin{abstract}
Progressive liver fibrosis is the primary cause of liver cirrhosis and hepatocellular carcinoma, and leads to considerable morbidity and mortality. Recent studies have demonstrated that microRNAs (miRNAs or miRs) are associated with fibrotic processes in liver disorders, although the exact role of miR-219 remains unclear and the relevant mechanisms remain to be completely understood. To the best of our knowledge, the present study was the first to demonstrate the functional implications of miR-219 expression during liver fibrosis. The present study reported that miR-219 exhibited significantly reduced expression in serum from patients and that its expression was negatively associated with clinical stage. It was also demonstrated that miR-219 attenuated angiotensin II-induced expression of pro-fibrotic markers, including $\alpha$-smooth muscle actin, atlastin GTPase 1 and collagen. Additionally, a $\mathrm{CCl}_{4}$-induced mouse liver injury model was used to demonstrate that miR-219 strongly suppressed liver fibrosis in vivo. Furthermore, the present study identified tumor growth factor $\beta$ receptor 2 (TGFBR2) as a direct target gene of miR-219. In conclusion, the results of the present study revealed that miR-219 may regulate pro-fibrotic markers by directly targeting the TGFBR2 gene and the miR-219/TGFBR2 signaling pathway may be a potential therapeutic target for liver fibrosis.
\end{abstract}

\section{Introduction}

Excessive fibrosis in the extracellular matrix (ECM) is the most important cause of liver fibrosis, and eventually results in liver injury (1). Hepatic stellate cells (HSCs) have been demonstrated to be the primary matrix-producing cells in the liver, serving a critical role in driving liver fibrosis

Correspondence to: Dr Hong-Liang Ou, Department of Liver Diseases, Ningbo No. 2 Hospital, 175 Yongfeng North Road, Ningbo, Zhejiang 315010, P.R. China

E-mail: ouhongliang6125@163.com

Key words: microRNA-219, tumor growth factor $\beta$ receptor 2, liver fibrosis, pro-fibrotic markers progression (2). It has been reported that any chronic stimulus may lead to the activation and growth of HSCs, which induce the accumulation of ECM proteins to trigger fibrosis and damage the liver tissue $(3,4)$. Although fundamental and genomic advances have contributed towards the understanding of the pathophysiology of the fibrosis response in the liver, definitive therapies are not fully accessible (5). Accordingly, there is a need for effective therapeutic strategies aimed at novel targets.

A previous study suggested that inflammatory cytokines are associated with fibrotic events and thus lead to the progression of liver disease (6). It is well established that tumor necrosis factor- $\alpha$, platelet-derived grow th factor and transforming growth factor- $\beta$ (TGF- $\beta$ ) may mediate a variety of inflammatory reactions and drive a progressive fibrotic response in liver disorders $(7,8)$. TGF- $\beta$ is known to be implicated in various processes associated with the development and progression of hepatic fibrogenesis. TGF- $\beta$ is composed of TGF- $\beta 1, \beta 2$ and $\beta 3$, which are able to modulate downstream signaling via TGF- $\beta$ receptor 1 (TGFBR 1$)$ and TGF- $\beta$ receptor 2 (TGFBR2) $(9,10)$. However, knowledge regarding the association between TGFBR 2 and hepatic fibrosis remains limited.

An increasing number of studies have demonstrated that miRNAs are involved in numerous molecular and cellular processes, serving an important role in the diagnosis and treatment of certain diseases (11). MicroRNAs (miRNAs or miRs), a class of short non-coding RNAs of 22 nucleotides in length, modulate gene expression post-translation or via the induction of mRNA degradation. A number of miRNAs have been demonstrated to be associated with fibrotic processes in liver disorders, including miR-29, miR-150 and miR-194 (12,13). Notably, the regulation of the expression of these miRNAs may halt fibrogenesis in vitro and in vivo, suggesting the significance of miRNAs as a potential target in fibrotic disease (14). However, the role of miR-219 in liver fibrosis remains to be completely characterized and the precise mechanisms remain unclear.

The present study aimed to investigate the implications of miR-219 expression during liver fibrosis in vitro and in vivo. Furthermore, the present study identified that miR-219 may directly target the TGFBR2 gene and that the miR-219/TGFBR2 signaling pathway may contribute to the diagnosis and treatment of fibrotic liver disease. 


\section{Materials and methods}

Clinical samples and cell lines. Tissues were obtained from 63 patients (37 males and 26 females; mean age, $61.9 \pm 7.9$ years) who were undergoing treatment at Ningbo No. 2 Hospital (Ningbo, China), in which a diagnosis of liver fibrosis was proven histopathologically following biopsy. The staging of fibrosis was as follows: 31 patients in stage $0-1 ; 20$ patients in stage $2-4$; and 12 patients in stage 5-6. In addition, 12 normal subjects (seven males and five females; mean age of $58.9 \pm 6.1$ years) were studied as controls. Blood samples were collected from all subjects, which were centrifuged $\left(1,000 \mathrm{x} \mathrm{g}, 10 \mathrm{~min}, 40^{\circ} \mathrm{C}\right)$ to harvest the separated serum, which was stored at $-80^{\circ} \mathrm{C}$ for further analysis. Written informed consent was obtained from either subjects or their family members, and the experiment was approved by the Ethics Committee of Ningbo No. 2 Hospital. In addition, HSCs were isolated from Sprague-Dawley rats $(n=24$; male; age, 12-16 weeks; weight, 450-550 g) purchased from the Chinese Academy of Science (Shanghai, China). These mice were housed five per cage under the following conditions: Constant temperature, $25^{\circ} \mathrm{C}$; humidity, $40-75 \% ; 12 \mathrm{~h}$ light/dark cycle; free access to food and water. HSCs were cultured in RPMI-1640 medium (cat. no. 11875093), supplemented with $10 \%$ calf serum (both Thermo Fisher Scientific, Inc., Waltham, MA, USA) in a humidified incubator with $5 \% \mathrm{CO}_{2}$ at $37^{\circ} \mathrm{C}$. After 48 h, angiotensin II (AngII; $100 \mathrm{nM}$; R\&D Systems, Inc., Minneapolis, MN, USA) was used to stimulate HSCs under additional incubation for $48 \mathrm{~h}$.

Reverse transcription-quantitative polymerase chain reaction $(R T-q P C R)$. Total miRNA was extracted using TRIzol ${ }^{\circledR}$ reagent (Invitrogen; Thermo Fisher Scientific, Inc.), according to the manufacturer's protocol. cDNA was synthesized using the cDNA RT kit (Applied Biosystems; Thermo Fisher Scientific, Inc.) at $42^{\circ} \mathrm{C}$. qPCR was performed using a SYBR-Green mix kit (Applied Biosystems; Thermo Fisher Scientific, Inc.) with a $20-\mu 1$ reaction system under conditions of $95^{\circ} \mathrm{C}$ for $30 \mathrm{sec}, 95^{\circ} \mathrm{C}$ for $3 \mathrm{sec}$ and $60^{\circ} \mathrm{C}$ for $30 \mathrm{sec}(40$ cycles). Relative expression levels of miRNA and other indicators were calculated using the $2^{-\Delta \Delta C q}(15)$ method in triplicate, and GAPDH or U6 were used as internal controls for normalization. The primer sequences used were as follows: miR-219 forward, 5'-ACACTCCAG CTGGGTGATTGTCCAAACGCAAT-3' and reverse, 5'-CTC AACTGGTGTCGTGGA-3; TGFBR2 forward, 5'-GTAGCT CTGATGAGTGCAATGAC-3' and reverse, 5'-CAGATATGG CAACTCCCAGTG-3'; collagen I forward, 5'-GAACCTGGG ATAGCAGGACAC-3' and reverse, 5'-CATAGTGGGTCC ACAAAGACATC-3'; collagen III forward, 5'-GAACCTGGG ATAGCAGGACAC-3' and reverse, 5'-CATAGTGGGTCC ACAAAGACATC-3'; $\alpha$-SMA forward, 5'-CATCACGAA CTGGGATGACATG-3' and reverse, 5'-CATCTTCTCCCT GTTGGCTTTAG-3'; FSP1 forward, 5'-GAGGCTTTACTC GCACTTCG-3' and reverse, 5'-ACCCTACGCAGACTCCCA G-3'; GAPDH forward, 5'-AGAAGGCTGGGGCTCArTTG-3' and reverse, 5'-AGGGGCCATCCACAGTCTTC-3'; and U6 reference gene forward, 5'-CTCGCTTCGGCAGCACA-3' and reverse, 5'-AACGCTTCACGAATTTGCGT-3.

miR-219 target prediction and luciferase reporter assay. The potential targets of miR-219 were predicted using online programs with databases of different algorithms, including EIMMO (http://www.mirz.unibas.ch/ElMMo3) and miRanda-mirSVR (http://microRNA.org). For the luciferase assay, HSCs $\left(2.5 \times 10^{5}\right.$ cell/well) were seeded in 6-well plates and co-transfected with luciferase reporter plasmids [pmiR-TGFBR2-wild-type (WT) or pmiR-TGFBR2-mutant (Mut)] (Promega Corporation, Madison, WI, USA), along with miR-219-mimics or controls using Lipofectamine ${ }^{\circledR} 2000$ (Gibco; Thermo Fisher Scientific, Inc.). Luciferase activity was analyzed using the Dual-Luciferase Reporter system (Promega Corporation), according to the manufacturer's protocol. The luciferase activity was determined via comparison with Renilla luciferase activity when the cells had been lysed with a passive lysis buffer.

Western blot analysis. Total protein was extracted from HSCs using a total protein extraction kit (ProMab Biotechnologies, Richmond, CA, USA), according to the manufacturer's protocol. Western blot analysis was performed as previously described (16). Primary antibody (rabbit-anti-p21; cat. no. ab188224, 1:500; rabbit-anti-GAPDH, cat. no. ab181603, 1:500; both Abcam, Cambridge, UK) were incubated at $4^{\circ} \mathrm{C}$ overnight, and the secondary goat anti-rabbit $\operatorname{IgG~H\& L}$ (horseradish peroxidase) antibody (cat. no. ab97051; 1:2,000; Abcam) was incubated at room temperature for $2 \mathrm{~h}$. GAPDH was used as a protein loading control.

Immunofluorescence cytochemistry. HSCs $\left(1.2 \times 10^{6}\right.$ cells $\left./ \mathrm{ml}\right)$ were respectively transfected with $30 \mathrm{nM}$ miRNA-219 mimics or negative control miRNA mimics in 6-well plates using siPort Neo-FX (both Ambion; Thermo Fisher Scientific, Inc.), according to the manufacturer's protocol. The following sequences were used: miR-219 mimic agomir sense, 5'-AAA AGA ATT CCC ACT TCCCAC TCC AGACATT- ${ }^{\prime}$ and antisense, 5'-AAAGCGGCCGCCCCTCACTTCTCCGTA ACCC-3'. The negative control for the agomir was sense, 5'-UUCUUCGAACGUGUCACGUTT-3' and antisense, 5'-ACGUGACACGUUCGGAGAATT-3'. Following transient transfection $(24 \mathrm{~h})$, the cells were synchronized in low-glucose medium (cat. no. 22320030; Thermo Fisher Scientific, Inc.) without serum for $24 \mathrm{~h}$, and stimulated with AngII, according to a previously-published protocol (17). The expression of $\alpha$-smooth muscle actin ( $\alpha$-SMA; cat. no. ab5831; 1:1,000; Abcam) was determined by immunofluorescence cytochemistry as previously described (18).

Chronic mouse liver injury model. $\mathrm{C} 57 \mathrm{BL} / 6 \mathrm{~J}$ mice $(\mathrm{n}=24$; male; age, 12-16 weeks; weight, 450-550 g) were housed five per cage under the following conditions: Constant temperature, $25^{\circ} \mathrm{C}$; humidity, $40-75 \%$; $12 \mathrm{~h}$ light/dark cycle; free access to food and water. These mice were administrated with $\mathrm{CCl}_{4}$ solution by intraperitoneal injection, and liver injury was established as previously described (19). The mice were randomly divided into three groups, termed the sham-operated group (8 mice), control group ( 8 mice with liver injury) and observation group [8 mice with liver injury receiving tail vein injection of miRNA-219 agonist $(5 \mathrm{mg} / \mathrm{kg} ; 200 \mu 1$,MedChemExpress, Monmouth Junction, NJ, USA)]. Mice were sacrificed following daily administration for 14 days, and liver tissues were collected and processed for detecting the expression of collagen mRNA by RT-qPCR, and for immunohistochemistry. All the procedures were performed 
A

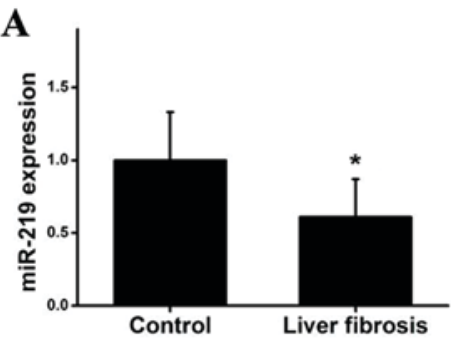

$\mathbf{C}$

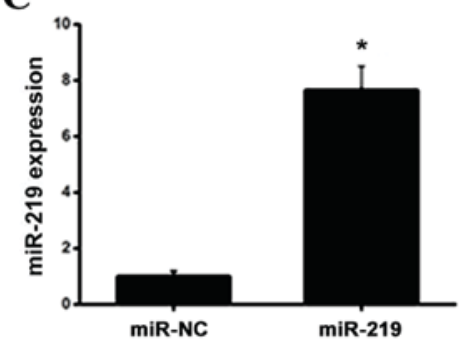

B

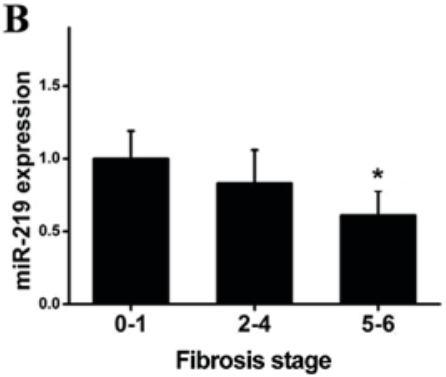

Figure 1. miR-219 expression is downregulated in patients with liver fibrosis. (A) miR-219 expression was differentially downregulated in the serum of patients with liver fibrosis. " $\mathrm{P}<0.05$ vs. control. (B) miR-219 expression was negatively associated with the clinical stage of liver fibrosis. * $\mathrm{P}<0.05$ vs. stage $0-1$. (C) Transfection with miR-219 was successful. ${ }^{*} \mathrm{P}<0.05$ vs. miR-NC. miR, microRNA; NC, negative control.

in accordance with national (D.L.n.26; March 4th, 2014) and international laws and policies (directive 2010/63/EU) (20). All experiments involving animals were approved by the Ethics Committee of Zhejiang Chinese Medical University.

Morphological alterations. Alterations in liver morphology were examined by Masson and hematoxylin and eosin (H\&E) staining. The liver tissues were fixed in $10 \%$ neutral formalin liquid for $24 \mathrm{~h}$ at room temperature, followed by fully-automatic dehydration, paraffin-embedding and slicing into tissue sections $(4 \mathrm{~mm})$. Serial liver slices were stained with Masson's trichrome stain (Beijing Solarbio Science \& Technology Co., Ltd., Beijing, China) for 10-20 min at room temperature, and H\&E for $45 \mathrm{~min}$ at room temperature.

Statistical analysis. Data were acquired from three independent experiments and are presented as the mean \pm standard deviation. Comparisons between two groups were performed using the Student's t-test, and a paired Student's t-test was used to analyze paired data. Comparisons among three or more groups were performed using one-way analysis of variance. Comparisons among three or more groups were performed with the Bonferroni correction used as a post hoc test. $\mathrm{P}<0.05$ was considered to indicate a statistically significant difference. The statistical analysis was performed using SPSS software (SPSS for Windows 17.0; SPSS Inc., Chicago, IL, USA).

\section{Results}

miRNA-219 is downregulated in patient serum. To investigate whether miRNA-219 serves a role in liver fibrosis, miRNA-219 expression in clinical blood samples was detected using RT-qPCR. As demonstrated in Fig. 1A, the expression of miR-219 was significantly decreased in the serum of patients when compared with the controls. To obtain further insight into the clinical relevance, the expression levels of miR-219 were compared in the blood samples of patients with various clinical pathological features. Notably, miR-219 was negatively associated with clinical stage, as demonstrated in Fig. 1B, and the transfection with miR-219 was successful (Fig. 1C). In summary, these data suggested that miRNA-219 may be involved in the development and progression of liver fibrotic responses.

TGFBR2 is verified as a direct binding target of miR-219. It has been reported that the TGFBR gene serves as a useful indictor associated with fibrotic events $(21,22)$. Using publicly accessible databases, the present study identified the putative miR-219 target sites in the 3'-UTR region of TGFBR2, as demonstrated in Fig. 2A. To further demonstrate whether TGFBR2 is a direct target of miR-219, the two putative miR-219 target sites (WT or Mut miR-219 target sequences) were fused into a luciferase reporter gene. Subsequently, HSCs were co-transfected with pmiR-WT or pmiR-Mut reporter and miR-219 mimics or controls, and cultured for $36 \mathrm{~h}$, prior to a luciferase reporter assay being performed. As demonstrated in Fig. 2B, cells transfected with miR-219 mimics exhibited significantly decreased luciferase activity from the WT 3'-UTR reporter gene, while treatment with miR-219 mimics failed to affect the luciferase activity of the mutant reporter gene. These observations indicated that miR-219 is able to directly bind to the 3'-UTR of TGFBR2. In order to further investigate whether miR-219 is able to successfully modulate TGFBR2 expression in HSCs, the subsequent experiments were performed to determine TGFBR2 expression in cells transfected with miR-219 mimics or controls. As demonstrated in Fig. 2C, the RT-qPCR results revealed that treatment with miR-219-mimics strongly attenuated TGFBR2 expression at the mRNA level compared with the controls. In line with this, western blot analysis indicated that cells transfected with miR-219 mimics exhibited significantly decreased TGFBR2 protein expression compared with the controls (Fig. 2D). These observations suggested that miR-219 may repress TGFBR2 expression at the mRNA and protein levels in HSCs by directly targeting the 3'-UTR of TGFBR2. 
A

Position 474-481 of TGFBR2 3 ' UTR $\quad 5^{\prime}$...ACAGCAUUAGCAUUUGACAAUCA...

hsa-miR-219a-5p

$3^{\prime}$

UCUUAACGCAAACCUGUUAGU

B

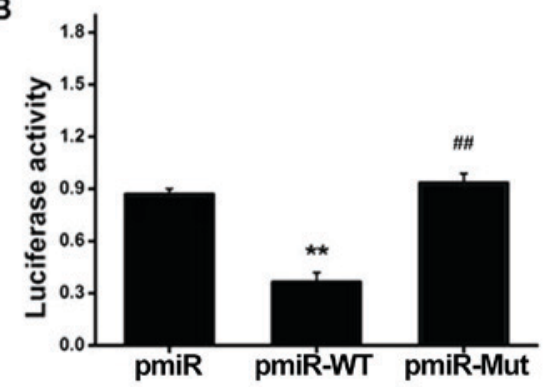

C

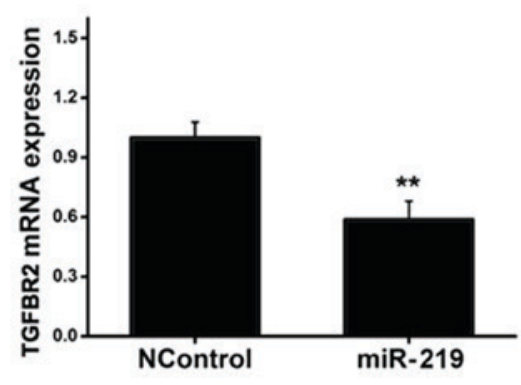

D

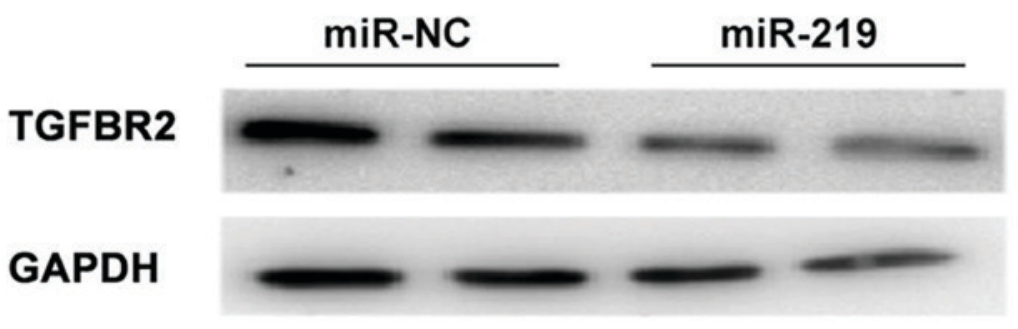

Figure 2. miR-219 regulates TGFBR2 expression by directly targeting the 3'-UTR of TGFBR2. (A) WT miR-219 target sites in the TGFBR2 3'-UTR. (B) Luciferase activity of the TGFBR2 3'-UTR reporter genes with miR-219-mimics. pmiR and pmiR-Mut exhibited no alteration in activity, while pmiR-WT exhibited an alteration compared with pmiR or pmiR-Mut. ${ }^{* *} \mathrm{P}<0.01$ vs. pmiR; ${ }^{\# \#} \mathrm{P}<0.01$ vs. pmiR-WT. (C) mRNA expression of TGFBR2 in HSCs transfected with miR-219-mimics using reverse transcription-quantitative polymerase chain reaction. ${ }^{* *} \mathrm{P}<0.01$ vs. NC. (D) TGFBR2 protein expression analysis in HSCs transfected with miR-219-mimics using western blotting. miR, microRNA; UTR, untranslated region; TGFBR2, tumor growth factor $\beta$ receptor 2; NC, negative control; WT, wild-type; Mut, mutant; HSCs, hepatic stellate cells.

miRNA-219 represses $\alpha$-SMA expression in vitro. Next, transfected HSCs were used to elucidate the functional significance of miRNA-219 in liver fibrosis. Ang II has been demonstrated to promote hepatic fibrogenesis and to upregulate miRNA expression in HSCs (23). To identify whether AngII exerts an effect on miR-219 expression during liver fibrotic disease, HSCs were exposed to AngII for $48 \mathrm{~h}$. RT-qPCR was used to examine miR-219 expression at the mRNA level, and the results revealed that miR-219 exhibited significantly decreased expression in response to AngII compared with the controls (Fig. 3A). Additionally, using immunofluorescence cytochemistry, $\alpha$-SMA expression was revealed to be increased following the activation of AngII in HSCs, whereas this response was reversed by treatment with miR-219 (Fig. 3B). These data indicated that miR-219 was able to downregulate AngII-induced $\alpha$-SMA expression in liver fibrosis.

miRNA-219 inhibits liver fibrosis by downregulation of pro-fibrotic markers. It is well known that the accumulation of $\alpha$-SMA, FSP1 and collagen can contribute toward fibrotic diseases $(24,25)$. To investigate the functional role of miR-219 in the liver fibrotic response, RT-qPCR was performed to determine the expression of $\alpha$-SMA, FSP1 and collagen I in response to miR-219. As demonstrated in Fig. 4, transfection with miRNA-219-mimics significantly attenuated the mRNA expression of $\alpha$-SMA, FSP1 and collagen I, compared with treatment with AngII alone. These data revealed that miR-219 exhibited an inhibitory effect in liver fibrosis via downregulation of pro-fibrotic markers.

miR-219 serves an important role in a mouse chronic liver injury model. An in vivo study was performed to obtain insight into the implications of miR-219 in the regulation of liver fibrosis in a mouse model. miRNA expression analysis was conducted from the liver tissues collected, and the results indicated that the mRNA expression of collagen type I and III was increased in mice treated with $\mathrm{CCl}_{4}$ (Fig. 5A and B). By contrast, miR-219 treatment markedly decreased the expression of the aforementioned pro-fibrotic indictors in a chronic mouse liver injury model (Fig. 5A and B), which was consistent with the in vitro study. Furthermore, histological examination revealed that treatment with $\mathrm{CCl}_{4}$ led to apparent lymphocyte infiltration and collagen deposition in liver tissues, whereas attenuation was identified in mice injected with miR-219, as presented in Fig. 5C. Taken together, these results suggested that miR-219 serves as an important agent in the control of collagen deposition during liver fibrosis in vivo.

\section{Discussion}

Hepatic fibrosis, which is regarded as a scarring process, is associated with an accumulated and altered deposition of ECM 
A

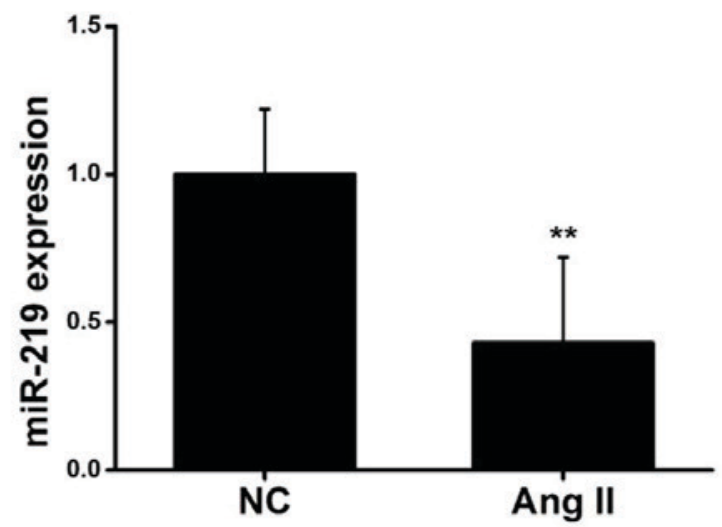

B
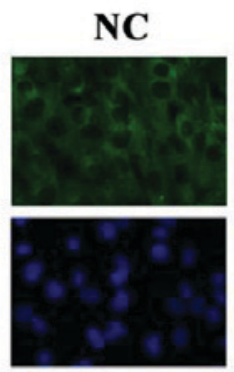

DAPI

a-SMA

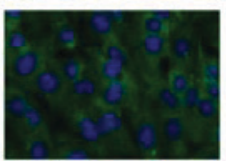

Ang II
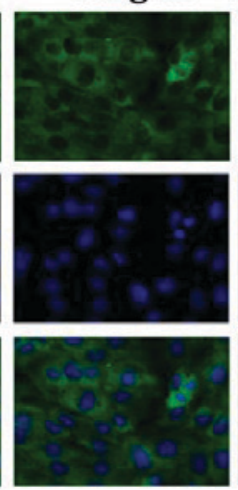

Ang II+miR-219
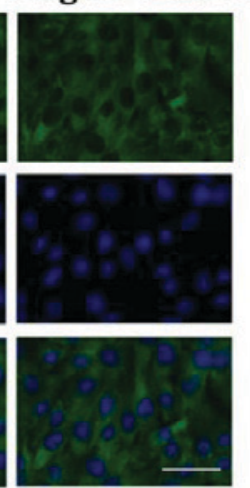

Figure 3. Increased expression of $\alpha$-SMA induced by AngII was weakened by miRNA-219 in vitro. (A) miRNA-219 expression was downregulated in HSCs treated with AngII by reverse transcription-quantitative polymerase chain reaction. ${ }^{* *} \mathrm{P}<0.01$ vs. NC. (B) $\alpha$-SMA expression stimulated with AngII and AngII + miRNA-181 was determined by immunofluorescence cytochemistry assay. miR, microRNA; AngII, angiotensin II; $\alpha$-SMA, $\alpha$-smooth muscle actin; HSCs, hepatic stellate cells (scale bar, $25 \mu \mathrm{m}$ ).

A

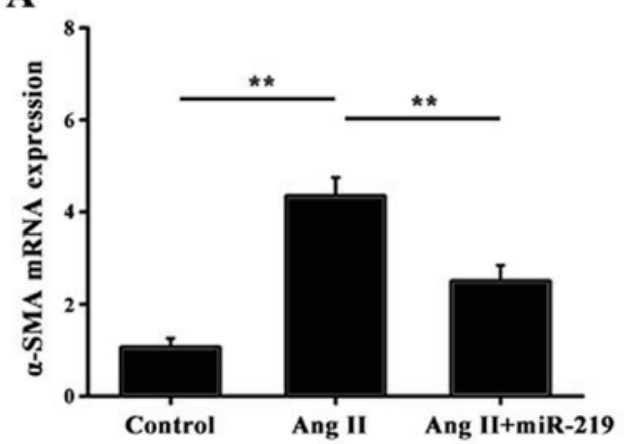

C

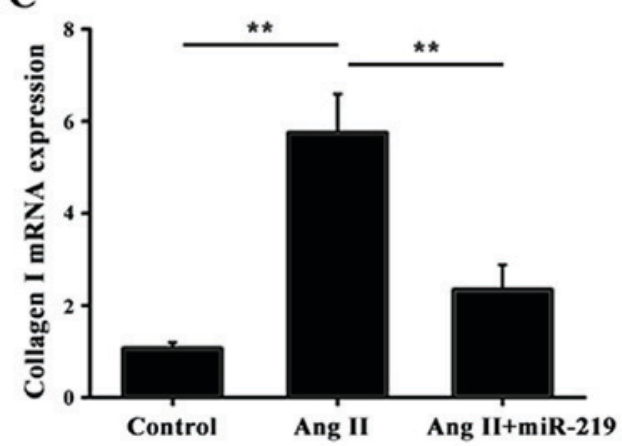

B

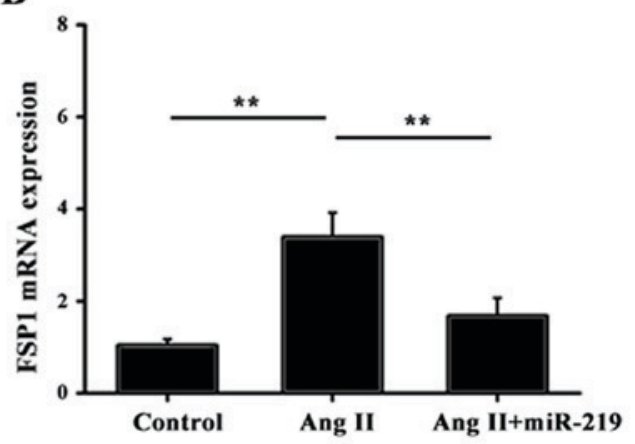

Figure 4. miRNA-219 inhibits liver fibrosis via downregulation of pro-fibrotic markers. The mRNA expression of (A) $\alpha$-SMA, (B) FSP1 and (C) collagen I was identified by reverse transcription-quantitative polymerase chain reaction in HSCs transfected with miRNA-219-mimics following the activation of AngII. ${ }^{* *} \mathrm{P}<0.01$. miR, microRNA; AngII, angiotensin II; $\alpha$-SMA, $\alpha$-smooth muscle actin; FSP1, atlastin GTPase 1.

in the liver. This progressive fibrotic response was characterized by cellular activation of HSCs, and aberrant expression of cellular factors and downstream molecule regulators (26). Recent studies have demonstrated that a certain number of miRNAs are involved in numerous cellular processes, including proliferation, migration, apoptosis, differentiation, the cell cycle and tumorigenesis (27). An increasing volume of evidence has revealed that a number of miRNAs function as critical regulators in alcoholic liver disease and fibrotic disorders. However, the role of miR-219 in liver fibrosis remains largely unknown. The present study reported on the functional relevance of miR-219 in liver fibrotic responses in vitro and in vivo. Notably, the present study provided evidence of the mechanism by which miR-219 may regulate pro-fibrotic markers implicated in liver fibrogenesis. Accordingly, it was demonstrated that miR-219 overexpression may contribute toward the diagnosis and treatment of liver fibrosis.

Little is known regarding the expression of miRNA-219 in liver fibrosis. A previous study demonstrated that miR-219 was potentially involved in the progression and metastasis of gastric cancer (28). miR-219 has also been demonstrated to be markedly attenuated in hepatocellular carcinoma, displaying a tumor-inhibitory role in hepatic carcinogenesis in HCC cell lines and tissues (29). Additionally, miR-219 suppressed 
A

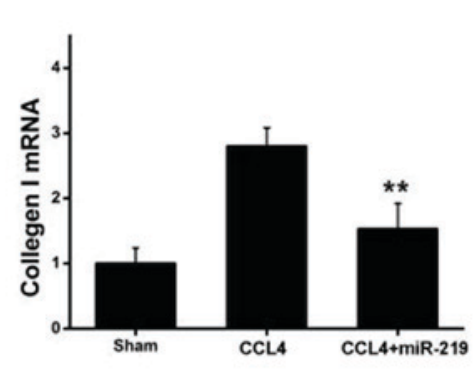

B

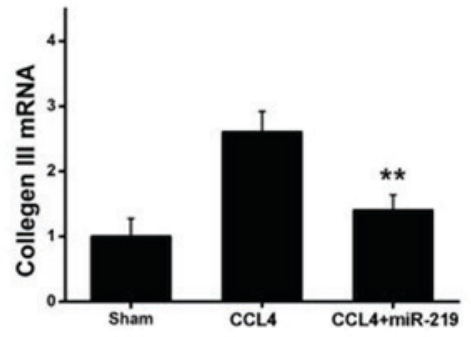

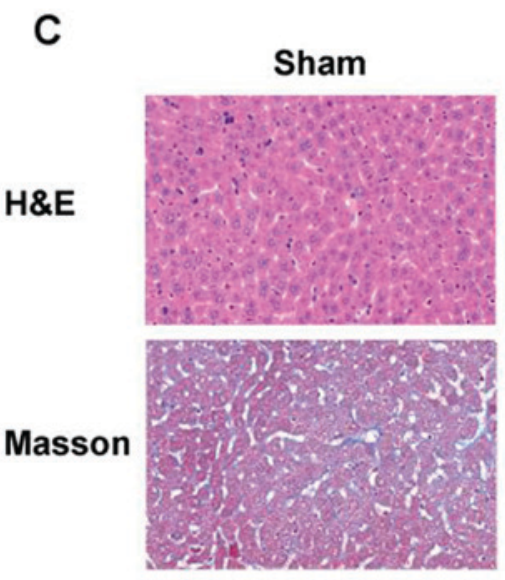

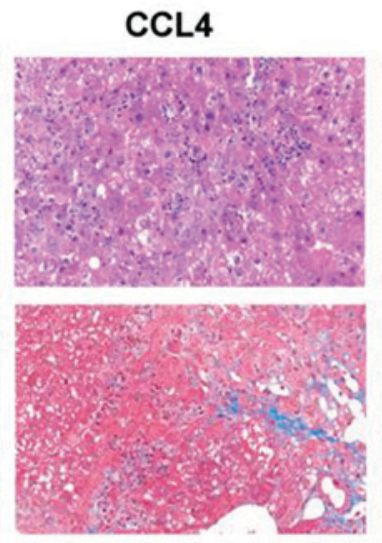

CCL4+miR-219

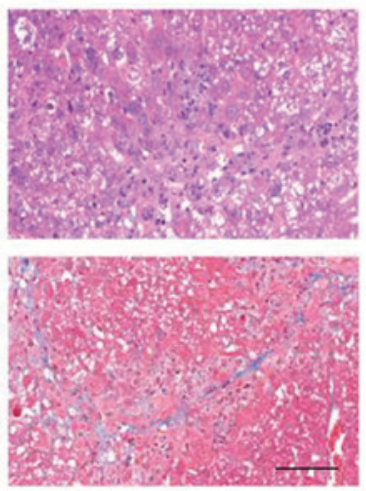

Figure 5. miR-219 inhibits the expression of pro-fibrotic markers and the fibrosis level in a chronic mouse liver injury model. The miRNA-219 agonist was delivered by tail vein injection in the model mice. The mRNA expression of (A) collagen type I and (B) collagen type III was downregulated in liver tissue following miR-219 treatment. (C) H\&E and Masson staining of liver tissues in mice. One-way analysis of variance was used in A and B. ${ }^{* *} \mathrm{P}<0.01$ vs. sham. miR, microRNA; H\&E, hematoxylin and eosin (scale bar, $25 \mu \mathrm{m}$ ).

the proliferation and growth of a variety of cells $(30,31)$. Notably, the present study identified an association between the expression of miR-219 and liver fibrosis. By analyzing the expression levels of serum miR-219 in clinical blood samples, it was revealed that the downregulation of miR-219 primarily occurred in patients with liver fibrosis, and that its expression is negatively associated with clinical stage, suggesting that miR-219 may be associated with the development and progression of liver fibrotic disease. Therefore, miR-219 was introduced for further investigation due to its expression signature.

It has been widely accepted that AngII is implicated in the pathogenesis of fibrotic response in kidneys, lungs and livers (32). Numerous studies have reported on AngII-mediated cardiac fibrosis through miRNA regulation. Siddesha et al (33) reported that AngII drives the migration of cardiac fibroblasts and thereby leads to cardiac fibrosis by inhibiting the expression of reversion inducing cysteine rich protein with kazal motifs, which is regarded as a critical agent for cardiac fibroblast migration (34). AngII also represses pro-apoptotic phosphatase and tensin homolog, and activates matrix metallopeptidase $2(35,36)$. In addition, a study undertaken by Ning et al (37) identified that treatment with AngII resulted in increased expression of miR-224 in adult rat cardiac fibroblasts. Ning et al (25) highlighted that AngII induced the NLR family pyrin domain containing 3 inflammasome/interleukin-1 $\beta$ axis and thus promoted HSC activation via the miR-21/sprouty RTK signaling antagonist 1/extracellular signal-regulated kinase/nuclear- $\kappa \mathrm{B}$ pathway. Based on these findings in fibrotic events, the present study investigated whether AngII may promote liver fibrosis via regulation of miR-219 and the precise mechanism involved in this. The present study revealed that AngII treatment decreased the expression of miR-219 in HSCs. However, overexpression of miR-219 decreased $\alpha$-SMA expression induced by AngII, suggesting that miR-219 exerted an important effect in HSCs via downregulation of this pro-fibrotic marker.

This notion was further supported by the results of in vitro and in vivo studies. Fibrotic markers, including $\alpha$-SMA, FSP1 and collagen, may be induced to participate in the signaling conduction pathway in order to promote fibrosis. Furthermore, a previous study (38) reported that TGF $\beta 1$ enhanced $\alpha$-SMA, COL1A1 and COL3A1 levels in order to drive renal fibrosis mediated by miR-433. Tian et al (39) reported that the apoptosis of hepatocytes induced by miR-34a markedly increased the expression of $\alpha$-SMA, TGF $\beta 1$ and collagen I at the mRNA and protein levels. The present study used RT-qPCR to determine the mRNA expression levels of specific liver fibrosis-associated molecules, including $\alpha$-SMA, FSP1 and collagen I in HSCs. Notably, it was demonstrated that miR-219 significantly downregulated the expression of $\alpha$-SMA, FSP1 and collagen I. Furthermore, a $\mathrm{CCl}_{4}$-triggered mouse liver fibrosis model was proposed to validate the functional relevance of miR-219 in liver fibrosis. Histological examination indicated that $\mathrm{CCl}_{4}$ induced apparent lymphocyte infiltration and collagen deposition in liver tissues, whereas miR-219 notably reversed this response by downregulating mRNA expression of collagen type I and III. These results emphasized the significance of miR-219 as a protective agent in liver fibrosis.

As described earlier, TGF- $\beta$ ligands contribute toward multiple biological processes, including cell proliferation, apoptosis, hypertrophy and mesangial cell fibrosis. TGF- $\beta$ ligand 
binding is known to promote the formation of TGFB2R dimers, and phosphorylation of threonine and serine residues activates TGFB1R. The activated TGF- $\beta$ receptor, which recruits the downstream signaling mediator, sequentially initiates a signal transduction that eventually modifies gene expression, thereby resulting in a fibrotic response (40). With regards to hepatic fibrogenesis, TGF- $\beta$ serves a key role in the differentiation and progression of HSCs, which may increase the accumulation and deposition of collagen, leading to progressive fibrosis and organ dysfunction (41). However, few studies have investigated the role of TGFB2R in liver fibrosis. To the best of our knowledge, the present study was the first to demonstrate that TGFB2R is regulated by miR-219 in liver fibrosis. A luciferase reporter assay indicated that miR-219 was able to directly bind to the 3'-UTR of TGFBR2, and further experiments demonstrated that transfection of miR-219 downregulated the expression of TGFBR2 at the mRNA and protein levels, suggesting TGFBR2 as a potential target of miR-219 implicated in fibrotic liver disease.

In conclusion, the present study provided a novel insight into the functional implications of miR-219 expression in the development and progression of liver fibrosis. It was also demonstrated that miR-219 overexpression served an inhibitory role in liver fibrosis by directly targeting TGFBR2. Therefore, the study provides a rationale for miR-219 as a promising biomarker for diagnosis and therapy of liver fibrosis.

\section{Acknowledgements}

Not applicable.

\section{Funding}

No funding was received.

\section{Availability of data and materials}

The datasets used and analyzed during the current study are available from the corresponding author on reasonable request.

\section{Authors' contributions}

LM designed the study, performed experiments, analyzed the data and wrote the manuscript. JM and H-LO performed the experiments. LM and H-LO analyzed the data and drafted the manuscript, designed and supervised the study, and edited the manuscript.

\section{Ethics approval and consent to participate}

Written informed consent was obtained from subjects, and the study was approved by the Ethics Committee of Ningbo No. 2 Hospital.

\section{Patient consent for publication}

Not applicable.

\section{Competing interests}

The authors declare that they have no competing interests.

\section{References}

1. Roderburg C, Urban GW, Bettermann K, Vucur M, Zimmermann H, Schmidt S, Janssen J, Koppe C, Knolle P, Castoldi M, et al: Micro-RNA profiling reveals a role for miR-29 in human and murine liver fibrosis. Hepatology 53: 209-218, 2011.

2. Elsharkawy AM, Oakley F and Mann DA: The role and regulation of hepatic stellate cell apoptosis in reversal of liver fibrosis. Apoptosis 10: 927-939, 2005.

3. Piscaglia F, Dudás J, Knittel T, et al: Expression of ECM proteins fibulin-1 and -2 in acute and chronic liver disease and in cultured rat liver cells. Cell Tissue Res 337: 449-462, 2009.

4. Yuan Y, Han Q, Li S, Tian Z and Zhang J: Wnt2b attenuates HSCs activation and liver fibrosis through negative regulating TLR4 signaling. Sci Rep 7: 3952, 2017.

5. Henderson $\mathrm{N}$ and Iredale J: Liver fibrosis: Cellular mechanisms of progression and resolution. Clin Sci (Lond) 112: 265-280, 2007.

6. He Y, Huang C, Zhang SP, Sun X, Long XR and Li J: The potential of microRNAs in liver fibrosis. Cell Signal 24: 2268-2272, 2012.

7. Kakino S, Ohki T. Nakayama H, Yuan X, Otabe S, Hashinaga T, Wada N, Kurita Y, Tanaka K, Hara K, et al: Pivotal role of TNF- $\alpha$ in the development and progression of nonalcoholic fatty liver disease in a murine model. Horm Metab Res 50: 80-87, 2018.

8. Ogawa S, Ochi T, Shimada H, Inagaki K, Fujita I, Nii A, Moffat MA, Katragadda M, Violand BN, Arch RH and Masferrer JL: Anti-PDGF-B monoclonal antibody reduces liver fibrosis development. Hepatol Res 40: 1128-1141, 2010.

9. Czochra P, Klopcic B, Meyer E, Herkel J, Garcia-Lazaro JF, Thieringer F, Schirmacher P, Biesterfeld S, Galle PR, Lohse AW and Kanzler S: Liver fibrosis induced by hepatic overexpression of PDGF-B in transgenic mice. J Hepatol 45: 419-428, 2006.

10. Ueberham E, Lã WR, Ueberham U, Schönig K, Bujard H and Gebhardt R: Conditional tetracycline-regulated expression of TGF-betal in liver of transgenic mice leads to reversible intermediary fibrosis. Hepatology 37: 1067-1078, 2003.

11. George J, Roulot D, Koteliansky VE and Bissell DM: In vivo inhibition of rat stellate cell activation by soluble transforming growth factor beta type II receptor: A potential new therapy for hepatic fibrosis. Proc Natl Acad Sci USA 96: 12719-12724, 1999.

12. Kato M and Natarajan R: MicroRNAs in diabetic nephropathy: Functions, biomarkers, and therapeutic targets. Ann N Y Acad Sci 1353: 72-88, 2015.

13. Zhong X, Chung AC, Chen HY, Dong Y, Meng XM, Li R, Yang W, Hou FF and Lan HY: miR-21 is a key therapeutic target for renal injury in a mouse model of type 2 diabetes. Diabetologia 56: 663-674, 2013

14. Huang Y, He Y and Li J: MicroRNA-21: A central regulator of fibrotic diseases via various targets. Curr Pharm Des 21: 2236-2242, 2015.

15. Livak KJ and Schmittgen TD: Analysis of relative gene expression data using real-time quantitative PCR and the 2(-Delta Delta C(T)) method. Methods 25: 402-408, 2001.

16. Zavadil J, Narasimhan M, Blumenberg $M$ and Schneider RJ: Transforming growth factor-beta and microRNA: mRNA regulatory networks in epithelial plasticity. Cells Tissues Organs 185: 157-161, 2007.

17. Wen M, Men R, Liu X and Yang L: Involvement of miR-30c in hepatic stellate cell activation through the repression of plasminogen activator inhibitor-1. Life Sci 155: 21-28, 2016.

18. Hyun J, Choi SS, Diehl AM and Jung Y: Potential role of Hedgehog signaling and microRNA-29 in liver fibrosis of IKK $\beta$-deficient mouse. J Mol Histol 45: 103-112, 2014.

19. Venugopal SK, Jiang J, Kim TH, Li Y, Wang SS, Torok NJ, $\mathrm{Wu} \mathrm{J}$ and Zern MA: Liver fibrosis causes downregulation of miRNA-150 and miRNA-194 in hepatic stellate cells, and their overexpression causes decreased stellate cell activation. Am J Physiol Gastrointest Liver Physiol 298: G101-G106, 2010.

20. Hartung T: Comparative analysis of the revised directive 2010/63/EU for the protection of laboratory animals with its predecessor 86/609/EEC-a t4 report. ALTEX 27: 285-303, 2010.

21. Müllenbach R, Fund N, Hall R, Dooley S and Lammert F: Genotype to phenotype: Modelling the impact of natural TGFbR 2 expression variation on fibrosis initiation in vivo. $Z$ Gastroenterol 49, 2011.

22. Zhang Y, Zhou X, Long YI, Peng S, Zhang Q and Mantian MI: Dihydromyricetin attenuates activation of hepatic stellate cells through TGF- $\beta 1 /$ Smad signaling pathway. J Third Mil Med Univ 40: 282-289, 2018. 
23. García-Sánchez O, López-Hernández FJ and López-Novoa JM: An integrative view on the role of TGF-beta in the progressive tubular deletion associated with chronic kidney disease. Kidney Int 77: 950-955, 2010

24. Border WA and Noble NA: Transforming growth factor beta in tissue fibrosis. N Engl J Med 331: 1286-1292, 1994.

25. Ning ZW, Luo XY, Wang GZ, Li Y, Pan MX, Yang RQ, Ling XG, Huang S, Ma XX, Jin SY, et al: MicroRNA-21 mediates angiotensin II-induced liver fibrosis by activating NLRP3 Inflammasome/IL-1 $\beta$ axis via targeting Smad7 and Spry1. Antioxid Redox Signal 27: 1-20, 2017.

26. Simms R, Coward WR, Pang L and Knox AJ; Carol Feghali-Bostwick: Identification of the sources of lung myofibroblasts using FSP1 And \pm -SMA As markers in idiopathic pulmonary fibrosis. Am J Respir Crit Care Med 181: A1117, 2010.

27. Ding H, Yang Q, Wang Z, et al: Effects of sulfotanshinone IIA sodium on murine renal interstitial fibrosis and CTGF level. Immunol J 27: 398-397, 2011.

28. Gressner AM and Weiskirchen R: Modern pathogenetic concepts of liver fibrosis suggest stellate cells and TGF-beta as major players and therapeutic targets. J Cell Mol Med 10: 76-99, 2006.

29. Kota J, Chivukula RR, O'Donnell KA, Wentzel EA, Montgomery CL, Hwang HW, Chang TC, Vivekanandan P, Torbenson M, Clark KR, et al: Therapeutic microRNA delivery suppresses tumorigenesis in a murine liver cancer model. Cell 137: 1005-1017, 2009.

30. Lei H, Zou D, Li Z, Luo M, Dong L, Wang B, Yin H, Ma Y, Liu C, Wang F, et al: MicroRNA-219-2-3p functions as a tumor suppressor in gastric cancer and is regulated by DNA methylation. PLoS One 8: e60369, 2013.

31. Huang N, Lin J, Ruan J, Su N, Qing R, Liu F, He B, Lv C, Zheng D and Luo R: MiR-219-5p inhibits hepatocellular carcinoma cell proliferation by targeting glypican-3. FEBS Lett 586: 884-891, 2012.

32. Wong TS, Liu XB, Wong YH, Ng RW, Yuen AP and Wei WI Mature miR-184 as potential oncogenic microRNA of squamous cell carcinoma of tongue. Clin Cancer Res 14: 2588-2592, 2008.
33. Siddesha JM, Valente AJ, Yoshida T, Sakamuri SS, Delafontaine P, Iba H, Noda M and Chandrasekar B: Docosahexaenoic acid reverses angiotensin II-induced RECK suppression and cardiac fibroblast migration. Cell Signal 26: 933-941, 2014.

34. Dugas JC, Cuellar TL, Scholze A, Ason B, Ibrahim A, Emery B, Zamanian JL, Foo LC, McManus MT and Barres BA: Dicerl and miR-219 are required for normal oligodendrocyte differentiation and myelination. Neuron 65: 597-611, 2010.

35. Xia Y, Jin X, Yan J, Entman ML and Wang Y: CXCR6 plays a critical role in angiotensin II-induced renal injury and fibrosis. Arterioscler Thromb Vasc Biol 34: 1422-1428, 2014.

36. Maegdefessel L, Azuma J, Toh R, Deng A, Merk DR, Raiesdana A, Leeper NJ, Raaz U, Schoelmerich AM, McConnell MV, et al: MicroRNA-21 blocks abdominal aortic aneurysm development and nicotine-augmented expansion. Sci Transl Med 4: 122ra22, 2012.

37. Ning $\mathrm{Q}$ and Jiang X: Angiotensin II upregulated the expression of microRNA-224 but not microRNA-21 in adult rat cardiac fibroblasts. Biomed Rep 1: 776-780, 2013

38. Lorenzen JM, Schauerte C, Hübner A, Kölling M, Martino F, Scherf K, Batkai S, Zimmer K, Foinquinos A, Kaucsar T, et al: Osteopontin is indispensible for AP1-mediated angiotensin II-related miR-21 transcription during cardiac fibrosis. Eur Heart J 36: 2184-2196, 2015.

39. Tian XF, Ji FJ, Zang HL and Cao H: Activation of the miR-34a/SIRT1/p53 signaling pathway contributes to the progress of liver fibrosis via inducing apoptosis in hepatocytes but not in HSCs. PLoS One 11: e0158657, 2016.

40. Santibañez JF, Quintanilla M and Bernabeu C: TGF- $\beta /$ TGF- $\beta$ receptor system and its role in physiological and pathological conditions. Clin Sci (Lond) 121: 233-251, 2011.

41. Liu Y: Epithelial to mesenchymal transition in renal fibrogenesis: Pathologic significance, molecular mechanism, and therapeutic intervention. J Am Soc Nephrol 15: 1-12, 2004.

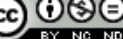

This work is licensed under a Creative Commons Attribution-NonCommercial-NoDerivatives 4.0 International (CC BY-NC-ND 4.0) License. 